\title{
Diferenciación temporal del perfil genético de la población de gatos de Cali, Colombia, en las últimas décadas
}

\author{
Andrés F. Peña-Cruz, Angie Patiño-Montoya* \\ Grupo de Investigación en Ecología Animal, Departamento de Biología, Facultad de Ciencias Naturales y Exactas, \\ Universidad del Valle, Cali, Colombia
}

\begin{abstract}
Resumen
En varios estudios se ha sugerido que variables como la intervención humana y el tiempo no generan cambios estadísticamente significativos en los perfiles genéticos de las poblaciones del gato doméstico, lo cual explicaría por qué la mayoría se encuentra en panmixia. Sin embargo, en pocos estudios se ha determinado el perfil genético de ciertas poblaciones más de dos veces en un lapso de varios años, por lo que no hay argumentos suficientes para concluir que el tiempo no afecta significativamente los perfiles de las poblaciones. Además, debe mencionarse que existen diferentes procesos estocásticos que actúan sobre cada uno de ellos. Ahora bien, la ciudad de Cali, Colombia, es una de las pocas en Latinoamérica en donde se ha establecido el perfil genético de los gatos en varias ocasiones (1999 y 2012). En el presente estudio se hizo un muestreo para establecer el perfil genético de dicha población durante el 2015. Se hicieron comparaciones mediante pruebas de chi cuadrado entre los periodos mencionados (1999 Vs. 2012, 1999 Vs. 2015 y 2012 Vs. 2015), y se estableció que no hubo cambios estadísticamente significativos entre ellos, aunque se registraron variaciones considerables en las frecuencias de los alelos White Spotting (S) y siamese $\left(c^{s}\right)$. Por otro lado, el árbol de distancias genéticas dendograma demostró que los perfiles de los años 2012 y 2015 fueron más similares en comparación con el de 1999. En este contexto, se sugiere que los efectos estocásticos, así como los de la selección humana y el factor tiempo son despreciables. C 2017. Acad. Colomb. Cienc. Ex. Fis. Nat.
\end{abstract}

Palabras clave: Gato doméstico; Perfil genético; Marcadores fenotípicos; Felis catus.

Temporal differentiation of the genetic profiles of the cat population in Cali, Colombia, during the last decades

\begin{abstract}
Several studies have suggested that variables such as human intervention and time do not generate statistically significant changes in the genetic profiles of domestic cat populations, which would explain why most are in panmixia. However, few studies have determined the genetic profile of certain populations more than twice over a period of several years, so there is insufficient evidence to conclude that time does not affected population profiles significantly. Also, it should be mentioned that there are different stochastic processes acting on each of these. The city of Cali, Colombia, is one of the few Latin American cat populations whose genetic profile has been established on several occasions (1999 and 2012). Therefore, in the present study we conducted a sampling to establish the genetic profile of this population during 2015. We compared the above-mentioned periods through chi-square tests, and we established that there were no statistically significant changes among them. However, there were considerable variations in the allele frequencies of White Spotting $(S)$ and siamese $\left(c^{s}\right)$. On the other hand, the genetic distances tree dendogram showed that 2012 and 2015 profiles were more similar than the 1999 profile. In this context, it is suggested that the stochastic effects, the human selection, and time were despicable. (C) 2017. Acad. Colomb. Cienc. Ex. Fis. Nat.
\end{abstract}

Key words: Domestic cat; Genetic profile; Phenotypic markers; Felis catus.

\section{Introducción}

El gato doméstico (Felis catus) ha sido un organismo modelo ampliamente usado en genética de poblaciones debido a la facilidad con la que pueden identificarse el genotipo y fenotipo de un grupo de genes de herencia mendeliana que determinan el color, patrón de coloración y largo del pelaje, además de algunas anormalidades esqueléticas (Lloyd, 1985; Ruiz-García, 1997; Kaelin y Barsh, 2013). A partir de estos se ha caracterizado el perfil genético de más de 400 poblaciones a nivel mundial y así mismo se ha permitido trazar diversos procesos de migración humana (Todd, 1977; Ruiz-García y Álvarez, 2000; Ruiz-García y Álvarez, 2008). Pese a lo mencionado anteriormente,

\footnotetext{
*Correspondencia:

Angie Patiño-Montoya, angie.patino@correounivalle.edu.co, angie. montoya317@gmail.com

Recibido: 19 de enero de 2017

Aceptado: 18 de abril de 2017
} 
algunos estudios como el de Clark (1975) muestran que la evidente preferencia humana por ciertos fenotipos de estos felinos podría modificar de manera artificial los perfiles de las poblaciones, lo cual lo llevó a sugerir que el gato doméstico no es un organismo modelo útil en genética de poblaciones. Sin embargo, el mismo Clark (1975) muestra que la mayoría de poblaciones estudiadas hasta el momento han demostrado estar en panmixia, lo cual podría contradecir el proceso de selección artificial. En este contexto se hace de interés determinar qué efecto tiene la preferencia humana de ciertos fenotipos y que procesos ocurren para mantener el estado panmíctico de las poblaciones. Además, otros estudios como el de Robinson (1985), Ruiz-García y Klein (1997) y Ruiz-García y Álvarez (2000) resaltan que la compleja estructura social de esta especie cosmopolita, junto con el evidente apareamiento aleatorio y el elevado flujo génico entre y dentro de poblaciones favorecen que el gato doméstico sea útil en estudios de genética poblacional.

En el caso de las poblaciones latinoamericanas, diversos estudios han demostrado un estado panmíctico en ellas, no siendo la excepción Colombia, cuyas 22 poblaciones urbanas de gatos caracterizadas (Ruiz-García y Álvarez, 1999; Peñuela y Cárdenas, 2012; Pardo, et al. 2014; Peña, et al. 2015; Montes-Díaz, et al. 2015; Pardo, et al. 2015; Peñuela, et al. 2016, Pardo, et al. 2016) han demostrado estar en panmixia y por ende en equilibrio Hardy-Weinberg según los análisis entre las frecuencias observadas y esperadas de los loci Orange y White Spotting, no obstante, un estudio realizado por Peña, et al. (2015) sugiere que a nivel microgeográfico existen procesos estocásticos que pueden modificar significativamente el perfil genético y con ello generar un desequilibrio $\mathrm{H}-\mathrm{W}$, el cual no necesariamente es causado por un apareamiento no aleatorio.

Ahora bien, el perfil genético de la población urbana de gatos en la ciudad de Cali ha sido caracterizado por RuizGarcía y Álvarez (1999), posteriormente fue caracterizado por Peña, et al. (2015) y Peñuela, et al. (2016) hacia el año 2012. Hasta el momento no se han reportado frecuencias de los alelos causantes de color café o "Brown", ni del patrón abisiano (Ticked abbysinian), ni del Manx o cola corta. Además, la población ha presentado un estado panmíctico, con poca variación en las frecuencias de los alelos mutantes de los loci Orange, Dilution, Agouti, Tabby, White spotting, Dominant White y Color repart (serie albina, incluye el patrón siamese). En este contexto, se hace interesante determinar si existen diferencias significativas en las frecuencias de estos alelos a través del tiempo, pues según como lo sugiere RuizGarcía y Álvarez (2008) los perfiles tienden a mantener su estabilidad genética en el caso de las poblaciones de gatos, reflejando frecuencias similares a las de las poblaciones origen. Con base en esto, el presente estudio ha determinado el perfil genético de la población urbana de gatos de Cali (Colombia) en el año 2015 con el objetivo de identificar si existen diferencias significativas entre las frecuencias de los años 1999, 2012 y las reportadas para el presente estudio.

\section{Materiales y métodos}

Marcadores fenotípicos. Siguiendo la nomenclatura propuesta por el Committee on Standardized Genetic Nomenclature for Cats (1968) se registraron un total de ocho loci: Orange vs. no-orange $(O, o)$; Agouti vs. no-agouti $(A, a)$; Tabby Mackerel o atigrado vs. Tabby Blotched $\left(t^{+}\right.$, $\left.t^{b}\right)$; Full color vs. dilution $(D, d)$; Pelo corto vs. Pelo largo o long hair $(L, l)$; White Spotting o manchado de blanco vs. no manchado $(S, s)$; Dominant White vs. silvestre (W, w) y Silvestre vs. siamese $\left(C, C^{s}\right)$. Cabe destacar que el locus Orange es ligado al sexo y su alelo dominante $(O)$ es epistático al patrón agouti (A), a su vez el no-agouti ( $a$ ) es epistático al patrón Tabby $\left(t^{+}, t^{b}\right)$ por otro lado, el alelo Dominant White $(W)$ es epistático frente al resto de loci relacionados con la coloración y patrón del pelaje.

Definición del área y estrategia de muestreo. Dentro del perímetro urbano de la ciudad de Cali (Colombia) se seleccionaron aleatoriamente un grupo de barrios con alta densidad de casas y de fácil acceso. En ellos se desarrolló el muestreo de los gatos durante el año 2015 mediante la observación directa de los individuos en las viviendas, tomando dato del sexo y determinando la presencia o ausencia de los diferentes alelos pertenecientes a los loci seleccionados para este estudio.

Determinación de frecuencias alélicas y equilibrio Hardy-Weinberg. Las frecuencias alélicas fueron estimadas asumiendo que existe equilibrio Hardy-Weinberg según la fórmula $\mathrm{p}^{2}+2 \mathrm{pq}+\mathrm{q}^{2}=1$, donde la frecuencia del alelo recesivo (q) de cada locus fue calculada como la raíz cuadrada del conteo de homocigotos recesivos de ese locus sobre el total de individuos, mientras que el dominante fue estimado como $\mathrm{p}=1-\mathrm{q}$. Ahora bien, dado que los loci Orange y White Spotting permiten la identificación de los fenotipos homocigotos y heterocigoto por observación directa, sólo es posible determinar si existe equilibrio Hardy-Weinberg en estos loci. Para ello se siguió la metodología de Christensen (2000) para el cálculo de las frecuencias genotípicas y de los valores de chicuadrado para probar la hipótesis de equilibrio $\mathrm{H}-\mathrm{W}$.

Análisis del perfil genético en tres periodos de tiempo. Para la comparación del perfil genético se usaron los alelos no-agouti (a), dilution $(d)$, Tabby blotched $\left(t^{b}\right)$, White $(W)$, White spotting $(S)$, siamese $\left(c^{s}\right)$, long hair $(l)$ y Orange $(O)$ en tres diferentes periodos de tiempo. El primer periodo, reportado por Ruiz-García y Álvarez (1999), representó el perfil genético del año 1999, el segundo reportado por Peña, et al. (2015) representó al año 2012, pues fue durante este periodo de tiempo que se realizó el muestreo de los individuos. Finalmente, el tercer periodo correspondió al muestreo realizado durante el año 2015 que es presentado en este estudio. Debe aclararse que no se tuvo en cuenta el estudio de Peñuela, et al. (2016) pese a que también reportó el perfil genético de la población de gatos de Cali, esto debido a que se estableció en el mismo año que se realizó el trabajo de Peña, et al. (2015), por lo que sería redundante para el análisis colocar dos perfiles pertenecientes al mismo 
año, además la metodología de muestreo usada por Peñuela, et al. (2016) no es totalmente comparable con la de RuizGarcía y Álvarez (1999) y Peña, et al. (2015).

Teniendo en cuenta lo anterior, se establecieron tres comparaciones (1999 vs. 2012 ; 1999 vs. $2015 ; 2012$ vs. 2015) en las que se realizó una prueba de chi-cuadrado para cada uno de los alelos previamente mencionados. De este modo cada una de las tres comparaciones de tiempo tuvieron 8 pruebas de chi-cuadrado referentes a los 8 alelos. Para cada prueba, se definió que la frecuencia esperada correspondía a la del año más antiguo, mientras que la frecuencia observada a la del año más reciente. Por otro lado, se estimaron las distancias genéticas entre los perfiles de los tres periodos de tiempo bajo el modelo de Nei (1972), graficándose un cluster con el algoritmo UPGMA en el programa MEGA®6 (Tamura, et al. 2013).

\section{Resultados}

Un total de 340 individuos fueron identificados en la población de Cali para el periodo de 2015. De los cuales 51,5\% (175 individuos) fueron hembras y 48,5\% (165 individuos) fueron machos, demostrándose una igualdad porcentual entre individuos de ambos sexos. Las frecuencias obtenidas para los alelos identificados en la población de Cali del 2015 se reportan en la Tabla 1. En ella se puede observar con respecto al periodo de tiempo inmediatamente anterior que no existe una variación notable en las frecuencias, con excepción del alelo White Spotting (S). En general, la comparación de 2012 vs. 2015 muestra un ligero aumento en la frecuencia del alelo dilution $(d)$, Tabby blotched $\left(t^{b}\right)$, Dominant White $(W)$ y así mismo se observa una ligera disminución en las frecuencias del patrón no-agouti $(a)$, siamese $\left(c^{s}\right)$, long hair $(l)$ y Orange $(O)$.

De otra parte, se demostró que las frecuencias observadas se ajustaron a las frecuencias esperadas para el locus Orange $\left(X^{2}=4,32 ; \mathrm{p}=0,12\right)$, lo cual sucedió también para el locus White Spotting $\left(X^{2}=2,49 ; \mathrm{p}=0,29\right)$, demostrándose a partir de ambos loci que la población de gatos domésticos de la ciudad de Cali del año 2015 se encuentra en equilibrio Hardy-Weinberg (ver tabla 2). En cuanto a los análisis de chi-cuadrado (tabla 3), debe mencionarse que ninguna frecuencia alélica ha mostrado cambios estadísticamente significativos entre los periodos de tiempo evaluados, sin embargo, hay diferencias considerables en la frecuencia del alelo White Spotting (S) entre los periodos 1999-2012 y 2012-2015, así como también se determinó con el alelo siamese $\left(c^{s}\right)$ entre 1999-2012. Los cambios más leves en las frecuencias se registraron para el no-agouti (a) durante 1999-2012 y 2012-2015, White Spotting (S) entre 19992015 y Dominant White $(W)$ entre 2012-2015.

El dendograma de distancias genéticas (figura 1) determinó que los perfiles de la población de gatos de Cali de los años 2012 y 2015 son bastante semejantes, lo cual es congruente con el hecho que son periodos de tiempo muy cercanos, mientras que el perfil genético del año 1999 forma una rama externa con el grupo 2012-2015. Estos resultados en conjunto con las comparaciones realizadas con chicuadrado muestran que evidentemente hay un cambio en las frecuencias alélicas de los tres periodos pese a que no sea estadísticamente significativo.

\section{Discusión}

En estudios de genética poblacional de gato doméstico, se asume que las diferencias en las frecuencias alélicas dentro y entre las poblaciones, son causadas por el hombre (Aguirre, 2007; Ruiz-García y Álvarez, 2000). La variación no significativa de las frecuencias alélicas encontradas en el presente trabajo, evidencia que las personas no influencian directamente la frecuencia de los caracteres fenotípicos; como se postula en la teoría de selección por novedad (RuizGarcía y Álvarez, 2000). Por el contrario, los resultados de las pruebas de chi-cuadrado, soportan la hipótesis de

Tabla 1. Frecuencias alélicas de ocho marcadores fenotípicos del pelaje en gato doméstico (F. catus) de la ciudad de Cali en tres años.

\begin{tabular}{cccc}
\hline & $\mathbf{1 9 9 9}$ & $\mathbf{2 0 1 2}$ & $\mathbf{2 0 1 5}$ \\
\cline { 2 - 4 } & $\mathbf{n = 2 8 0}$ & $\mathbf{n = 2 6 3}$ & $\mathbf{n = 3 4 0}$ \\
\hline $\mathbf{a}$ & 0,8390 & 0,7449 & 0,7227 \\
$\mathbf{d}$ & 0,4710 & 0,3273 & 0,4060 \\
$\mathbf{t}^{\mathbf{b}}$ & 0,1800 & 0,2329 & 0,2616 \\
$\mathbf{W}$ & 0,0054 & 0,0153 & 0,0178 \\
$\mathbf{S}$ & 0,3190 & 0,6531 & 0,3411 \\
$\mathbf{c}^{\mathbf{s}}$ & 0,2690 & 0,4383 & 0,3865 \\
$\mathbf{l}$ & 0,3300 & 0,4834 & 0,4537 \\
$\mathbf{O}$ & 0,1930 & 0,2481 & 0,2294 \\
\hline
\end{tabular}

$\mathrm{n}=$ número de muestras $a=$ Agouti, $d=$ dilution, $t=$ tabby blotched, $W=$ dominant white, $S=$ white spotting, $C=$ Colour repart, $l=$ long hair $O=$ orange

Tabla 2. Demostración de equilibrio $\mathrm{H}-\mathrm{W}$ calculado para los locus Orange (O) y White Spotting (S) en la población de gatos domésticos (F. catus) en la ciudad de Cali para el año 2015.

\begin{tabular}{lcccc}
\hline Locus & Fenotipo & Observado & Esperado & $\boldsymbol{X}^{\mathbf{2}}$ \\
\hline Orange & Oy & 41 & 36,02 & 0,69 \\
$\mathrm{n}=327$ & oy & 116 & 120,98 & 0,21 \\
& OO & 12 & 8,95 & 0,73 \\
& Oo & 49 & 60,10 & 2,05 \\
& oo & 109 & 100,95 & 0,64 \\
& & & Total & 4,32 \\
& & & $\boldsymbol{p}$ & 0,12 \\
\hline White & SS & 31 & 37,35 & 1,08 \\
Spotting & Ss & 157 & 144,29 & 1,12 \\
n= 321 & ss & 133 & 139,36 & 0,29 \\
& & & Total & 2,49 \\
& & & $\boldsymbol{p}$ & 0,29 \\
\hline
\end{tabular}

n: número de muestras, p: valor de significancia estadística. 
Tabla 3. Valores de chi-cuadrado $\left(X^{2}\right)$ y p-valor obtenidos por cada frecuencia alélica en las tres comparaciones definidas para los tres periodos de tiempo.

\begin{tabular}{ccccccc}
\hline & \multicolumn{2}{c}{$\mathbf{1 9 9 9 - 2 0 1 2}$} & \multicolumn{2}{c}{$\mathbf{1 9 9 9 - 2 0 1 5}$} & \multicolumn{2}{c}{$\mathbf{2 0 1 2 - 2 0 1 5}$} \\
\cline { 2 - 7 } & $\boldsymbol{X}^{\mathbf{2}}$ & $\mathbf{p}$-valor & $\boldsymbol{X}^{\mathbf{2}}$ & $\mathbf{p}$-valor & $\boldsymbol{X}^{\mathbf{2}}$ & p-valor \\
\hline $\mathbf{a}$ & 0,01 & 0,92 & 0,02 & 0,90 & 0,00 & 0,98 \\
\hline $\mathbf{d}$ & 0,04 & 0,83 & 0,01 & 0,92 & 0,02 & 0,89 \\
$\mathbf{t}^{\mathbf{b}}$ & 0,02 & 0,90 & 0,04 & 0,85 & 0,00 & 0,95 \\
$\mathbf{W}$ & 0,02 & 0,89 & 0,03 & 0,87 & 0,00 & 0,98 \\
$\mathbf{S}$ & 0,35 & 0,55 & 0,00 & 0,97 & 0,15 & 0,70 \\
\hline $\mathbf{c}^{\mathbf{s}}$ & 0,11 & 0,74 & 0,05 & 0,82 & 0,01 & 0,94 \\
$\mathbf{l}$ & 0,07 & 0,79 & 0,05 & 0,83 & 0,00 & 0,97 \\
\hline $\mathbf{O}$ & 0,02 & 0,90 & 0,01 & 0,93 & 0,00 & 0,97 \\
\hline
\end{tabular}

$a=$ Agouti, $d=$ dilution, $t=$ tabby blotched, $W=$ dominant white, $S=$ white spotting, $C=$ Colour repart, $l=$ long hair, $O=$ orange

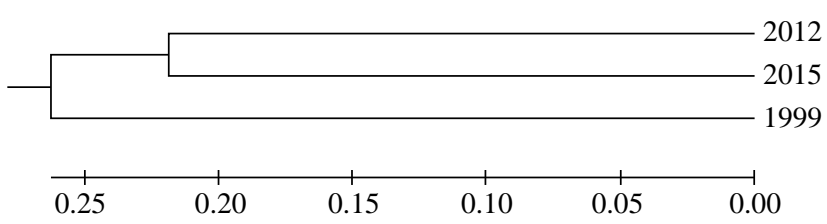

Figura 1. Dendograma UPGMA generado a partir de las distancias genéticas de Nei (1972) obtenidos a partir del perfil genético de tres diferentes periodos de tiempo (1999-2012-2015) de la población urbana de gatos de la ciudad de Cali.

estabilidad genética, en la cual los perfiles genéticos de las poblaciones se mantienen constantes en el tiempo (RuízGarcía y Álvarez, 2008). Por lo tanto, los cambios aparentes en las frecuencias y lo observado en el dendrograma UPGMA, son resultado de procesos estocásticos ajenos a la intervención humana.

La conservación del perfil génico a pesar de un "aparente" proceso de selección artificial, se puede deber a la combinación de dos factores: la historia natural de la especie y la costumbre de cría del gato doméstico por parte de la sociedad Caleña. Estos factores estarían altamente correlacionados con el concepto de selección por novedad de Todd (1977). En cuanto a la estructura social de la especie, debe decirse en primer lugar que los machos tienen territorios de mayores tamaños que las hembras, siendo estas últimas las que exhiben un mayor grado de dispersión. En segundo lugar, la jerarquía de los machos se encuentra asociada a la estructura antropogénica donde se encuentren (Horn, et al. 2011). Además, la posibilidad que una hembra sea fecundada simultáneamente por varios machos, dada la promiscuidad de ambos sexos y el apareamiento entre parientes (Natoli y De Vito, 1991; Say, et al. 1999) puede facilitar un alto grado de dispersión de diferentes caracteres genéticos.

En cuanto a la costumbre de cría en la ciudad, debe mencionarse que el 50\% de la población del gato doméstico de Cali se encuentra en los estratos bajos, los cuales presentan una mayor fecundidad respecto al estrato alto y el promedio general de la ciudad, también es el grupo de la población que cuenta con una menor cantidad de individuos esterilizados (Cooperativa de Trabajo Asociado Prestadores de Servicios Agrupados, 2011). Esto sería un indicativo que la mayor parte de la población de estos felinos no se encuentra sometida a selección artificial, y el aporte que realizan los gatos de raza seleccionados no es significativo en la escala temporal del presente estudio.

En conclusión, las frecuencias alélicas de la población de gatos en la ciudad de Cali, no variaron de manera significativa en las últimas dos décadas debido al bajo control y selección de individuos por parte de las personas de la ciudad, que favorecen más a la especie para mantenerla en panmixia que bajo presiones de selección artificial. Aún existe un vacío en el conocimiento, que permita determinar el grado de selección artificial al que se pueden encontrar sometidas las grandes poblaciones de gatos urbanas Latinoamericanas, como parece postularse en todos los estudios de la variación genética del gato doméstico adelantados en estos lugares.

\section{Agradecimientos}

Los autores agradecen a Stefania Cerón Realpe, Ingrid Jhoana López y Tatiana Bolaños por la colaboración brindada en el proceso de muestreo de los gatos durante el año 2015. También agradecemos a la Universidad del Valle y los dueños de las mascotas quienes permitieron que el registro de las mascotas fuera posible.

\section{Conflicto de intereses}

No se presenta conflicto de intereses.

\section{Referencias}

Aguirre, E. (2007). Flujo génico: métodos para estimarlo y marcadores moleculares. En L. E. Eguiarte, V. Souza, X. Aguirre (ed.). Ecología molecular. (pp. 49-61). México: Universidad Nacional Autónoma de México.

Christensen, A. (2000). Cats as an aid to teaching genetics. Genetics, 155 (3): 999-1004.

Clark, J. (1975). The effects of selection and human preference on coat colour gene frequencies in urban cats. Heredity, 35 (2): 195-210.

Committee on Standardized Genetic Nomenclature for Cats. (1968). Standardized genetic nomenclature for the domestic cat. Journal of Heredity, 59: 39-49.

Cooperativa de Trabajo Asociado Prestadores de Servicios Agrupados. (2011). Estudios de factibilidad para el centro de vigilancia y control de zoonosis y de protección animal del municipio de Cali. Zoonosis. Recuperado de http:// calisaludable.cali.gov.co/saludPublica/2012-Zoonosis/ Documento_Centro_de_Zoonosis_Final.pdf

Horn, J.A., Mateus, N., Warner, R.E., \& Heske, J.E. (2011). Home range, habitat use, and activity patterns of freeroamings domestics cats. Journal of Wildlife Management, 75 (5): 1177-1185.

Kaelin, C., \& Barsh, G. (2013). Genetics of pigmentation in dogs and cats. Annual Review of Animal Biosciences, 1 (1): $125-156$. 
Lloyd, A. (1985). Geographic distribution of mutant alleles in domestic cat populations of New England and the Canadian Maritimes. Journal of Biogeography, 12 (4): 315-322.

Montes-Díaz, Y., Cardales-Barrios, Y., \& Pardo-Pérez, E. (2015). Análisis de la variabilidad genética de las poblaciones de gatos domésticos (Felis catus) mediante genes del pelaje en Cartagena, Colombia. Revista de la Academia Colombiana de Ciencias Exactas Físicas y Naturales, 39 (153): 520-526.

Natoli, E., \& De Vito, E. (1991). Agonistic behaviour, dominance rank and copulatory success in a large multi-male feral cat colony (Felis catus L.) in central Rome. Animal Behaviour, 42 (2): 227-241.

Nei. M. (1972). Genetic distance between populations. The American Naturalist, 106 (949): 283-292.

Pardo, E., Morales, J., \& Cavadia, T. (2014). Estudio de la diversidad genética de la población de gato doméstico (Felis catus) en Monteria, Colombia. Bistua, 12 (2): 35-47.

Pardo, E., Causil, L., \& Rodríguez, A. (2015). Estudio de la diversidad genética de gato doméstico (Felis catus) mediante genes asociados al color del pelaje en Lorica-Córdoba, Colombia. Archivos de Zootecnia, 64 (248): 389-395.

Pardo, E., Montes, Y., \& Cardales, Y. (2016). Variabilidad genética del gato doméstico (Felis catus) en Magangué, Bolívar, Colombia. Revista de Investigaciones Veterinarias del Perú, 27 (2): 277-287.

Peña-Cruz, A.F., Sandoval Arango, S., Patiño Montoya, A., Bedoya, M., Rodríguez, A., Orjuela, J., Ortega, A., López, J., Molina Henao, E., Guzmán, A., Gil, J., \& Cárdenas Henao, H. (2015). Análisis genético de la población de gatos del norte y sur de Cali, Colombia. Acta Biológica Colombiana, 20 (1): 111-118.

Peñuela, M., \& Cárdenas, H. (2012). Marcadores genéticos del pelaje en gatos domésticos de Capurganá-Colombia. Momentos de Ciencia, 9 (1): 28-33.

Peñuela, M., Pardo, E., García, V.H., \& Cárdenas, H. (2016). Marcadores genéticos del pelaje del gato doméstico Felis catus (Felidae) del suroccidente colombiano. Revista MVZ Córdoba, 21 (2): 5390-5403.
Robinson, R. (1985). The domestic cat in genetic research. Trends in Genetics, 1 (1): 236-239.

Ruiz-García, M. (1997). Genetic relationships among some new cat populations sampled in Europe: a spatial autocorrelation analysis. Journal of Genetics, 76 (1): 1-24.

Ruiz-García, M., \& Klein, K. (1997). Genetic structure of populations of the domestic cat in Catalonia (Spain) and upper Midwestern USA: a microgeographic and macrogeographic study. Journal of Genetics, 76 (2): 99-115.

Ruiz-García, M., \& Álvarez, D. (1999). Análisis filogenético de 21 poblaciones latinoamericanas de gatos mediante 10 loci morfológicos, utilizando métodos de matrices de distancias genéticas y de máxima parsimonia. Boletín de la Real Sociedad Española de Historia Natural (Sección Biológica), 95 (3-4): 139-164.

Ruiz-García, M., \& Álvarez, D. (2000). Genetic microstructure in two spanish cat populations. I: genic diversity, gene flow and selection. Genes \& Genetic Systems, 75 (2): 269-280.

Ruiz-García, M., \& Álvarez, D. (2008). A biogeographical population genetics perspective of the colonization of cats in Latin America and temporal genetic changes in Brazilian cat populations. Genetics and Molecular Biology, 31 (3): 772-782.

Say, L., Pontier, D., \& Natoli, E. (1999). High variation in multiple paternity of domestic cats (Felis catus L.) in relation to environmental conditions. Proceedings of the Royal Society of London B: Biological Sciences, 266 (1433): 2071-2074.

Tamura, K., Stecher, G., Peterson, D., Filipski, A., \& Kumar, S. (2013). MEGA 6: Molecular evolutionary genetics analysis version 6.0. Molecular Biology and Evolution, 30 (12): 2725-2729.

Todd, N. (1977). Cats and commerce. Scientific American, 237 (1): 100-107. 